\title{
HYPERBOLIC WAVELET LEADERS FOR ANISOTROPIC MULTIFRACTAL TEXTURE ANALYSIS
}

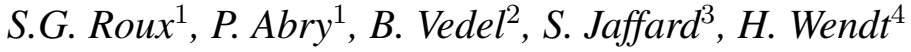 \\ ${ }^{1}$ Physics Dept., CNRS (UMR 5672), Ecole Normale Supérieure de Lyon, Université de Lyon, France. \\ ${ }^{2}$ LMBA, CNRS (UMR 6205), Université de Bretagne, Vannes, France. \\ ${ }^{3}$ LAMA, CNRS (UMR 8050), Université Paris-Est Créteil, France \\ ${ }^{4}$ IRIT-ENSEEIHT, CNRS (UMR 5505), Université de Toulouse, France.
}

\begin{abstract}
Scale invariance has proven a crucial concept in texture modeling and analysis. Isotropic and self-similar fractional Brownian fields $(2 \mathrm{D}-\mathrm{fBf})$ are often used as the natural reference process to model scale free textures. Its analysis is standardly conducted using the 2D discrete wavelet transform. Generalizations of $2 \mathrm{D}-\mathrm{fBf}$ were considered independently in two respects: Anisotropy in the texture is allowed while preserving exact self-similarity, analysis then needs to be conducted using the 2D-Hyperbolic wavelet transform ; Multifractality enables more versatile scale free models but requires isotropy, analysis is then achieved using wavelet leaders. The present paper proposes a first unifying extension, which is enabled through the following two key contributions: The definition of 2D process that incorporates jointly anisotropy and multifractality : The definition of the corresponding analysis tool, the hyperbolic wavelet leaders. Their relevance are studied by numerical simulations using synthetic scale free textures.
\end{abstract}

Index Terms - Texture, Multifractality, Anisotropy, Hyperbolic wavelet leaders

\section{INTRODUCTION}

Context. Scale free properties, or scale invariance, is often regarded as a central concept in real-world texture modeling (cf. e.g., [1-9]). Fractional Brownian fields (2D-fBf), because of their conceptual and practical simplicity and because of their being natural isotropic 2D extension of 1D fractional Brownian motion [10], are massively used in theory and applications as reference processes to model scale free textures. 2D-fBf are isotropic, Gaussian exactly self-similar processes. Their dynamics are thus controlled by the unique so-called self-similarity parameter $0<H<1$.

For practical analysis, it is nowadays well-accepted that wavelet transforms are ideal tools to evidence scale free properties and to estimate the corresponding scaling parameters $[11,12]$. Notably, it is well documented that the moments

Work supported by French ANR BLANC 2011 AMATIS BS0101102. of the coefficients of a 2D-Discrete Wavelet Transform (2DDWT), $d_{X}(j, \mathbf{k})$, computed on a 2D-fBf, follow, across analysis scales $a=2^{j}$, a power-law behavior with scaling exponent controlled by $H, \mathbb{E}\left|d_{X}(j, \mathbf{k})\right|=C 2^{2 j H}$, thus easily permitting to reveal isotropic self-similarity and to estimate $H$ (cf. e.g., [11]).

However, as a modeling paradigm, 2D-fBf suffers from two major limitations: i) Scale free properties are governed by a single scaling exponent $H$, which excludes the use of multifractality (with a collection of scaling exponents) to better account for the richness of scaling encountered in real-world textures [2-6] ; ii) Anisotropy is not permitted in the classical definition of 2D-fBf while it is another natural and important property of texture [1].

Related works. These two restrictions have been addressed independently. Multifractal processes $[13,14]$ are often used as versatile models permitting to enrich scale free properties by allowing fluctuations along space of the local regularity of the process and thus requiring a collection of Hölder exponents $h$ to account for scaling in textures instead of one single parameter $H$, a richness quantified by the so-called multifractal spectrum $D(h)[1,2,11,14]$. These richer scaling properties are obtained though at the price of maintaining isotropy. The relevant analysis of multifractality in textures requires the replacement of the 2D-DWT coefficients, by wavelet leaders, consisting of local hierarchical suprema of such coefficients [11]. Independently, anisotropy has been been married with scale free properties in Operator Scaling Gaussian Random Fields (OSGRF) processes [15]. These anisotropic Gaussian processes are exactly self-similar, which precludes multifractal extensions. It has recently been shown that the use the 2D-hyperbolic wavelet transform (2D-HWT) [16] enables a theoretically sound and practically efficient joint estimation of both self-similarity and anisotropy [17]. However, there has been so far no attempt to propose models accommodating both anisotropy and multifractality in textures, or to devise the corresponding analysis tools.

Goals, contributions and outline. The present contribution has thus a double aim: First, a candidate model for anisotropic 
multifractal texture will be defined and described (Section 2); Second, a tool for the joint analysis of anisotropy and multifractality, the Hyperbolic wavelet leaders, will be proposed (Section 3). The relevance of the proposed model and analysis tools will be illustrated and assessed using synthetic scalefree textures (Section 4).

\section{ANISOTROPIC MULTIFRACTAL TEXTURES}

Definition. Combining the definition of multidimensional isotropic multifractal random fields, introduced in [13] as a natural extension of univariate multifractal random walks (cf. e.g., [18]), with the definition of of OSGRF in [15], an anisotropic extension of 2D-fBf, we propose the following original operational definition of anisotropic multifractal textures

$$
X_{\alpha_{0}, H_{0}, \lambda_{0}}(\mathbf{x})=\int_{\mathbb{R}^{2}} \frac{\left(e^{i\langle\mathbf{x}, \boldsymbol{\xi}\rangle}-1\right)}{\|\boldsymbol{\xi}\|_{2, \alpha_{0}}^{\left(H_{0}+1\right)}} G_{\lambda_{0}}(\boldsymbol{\xi}) d \boldsymbol{\xi}
$$

where $\mathbf{x}=\left(x_{1}, x_{2}\right)$ and $\boldsymbol{\xi}=\left(\xi_{1}, \xi_{2}\right)$ denote respectively the space and Fourier domains coordinates. This toy-model is constructed to incorporate the three-properties targeted here: Parameter $0<H_{0}<1$ controls the global scale free property of the process via the fractional intergration kernel $\|\boldsymbol{\xi}\|_{2, \alpha_{0}}^{\left(H_{0}+1\right)}$; Parameter $\lambda_{0}>0$, referred to as the intermittency or multifractality parameter, controls the degree of variation of the local regularity along space, via the intermediate process $G_{\lambda_{0}}(\boldsymbol{\xi})$ defined as the Fourier transform of $g(\mathbf{x})=e^{\omega(\mathbf{x})} d W(\mathbf{x})$, where $d W(\mathbf{x})$ correspond to a 2D-Wiener measure and $\omega(\mathbf{x})$ to an independent Gaussian stationary process whose covariance is chosen of the form

$$
\operatorname{cov}(\omega(\mathbf{x}), \omega(\mathbf{y}))=-\lambda_{0}^{2} \log \left(\|\mathbf{x}-\mathbf{y}\|_{2}\right),
$$

to ensure scale-free properties ; Parameter $0<\alpha_{0}<2$ controls the global anisotropy via the use of an anisotropic pseudo-norm $\|\boldsymbol{\xi}\|_{2, \alpha_{0}}=\sqrt{\left|\xi_{1}\right|^{2 / \alpha_{0}}+\left|\xi_{2}\right|^{2 /\left(2-\alpha_{0}\right)}}$ in the fractional integration kernel.

Setting $\alpha_{0} \equiv 1$ obviously permits to recover the isotropic case and thus corresponds to the isotropic multifractal random fields in [13]. While $\lambda_{0}=0$ is in principle excluded from the definition, we will by convention set $\omega(\mathbf{x}) \equiv 1$ for $\lambda_{0}=0$. In this case, one recovers the classical exactly self-similar Gaussian OSGRF defined in [15]. Setting jointly $\alpha_{0} \equiv 1$ and $\lambda_{0} \equiv 0$ obviously yields the simple Gaussian isotropic exactly self-similar 2D-fBf.

Anisotropic multifractal analysis. Elaborating on isotropic multifractal analysis, we propose here to characterize scalefree properties by an anisotropic multifractal spectrum, defined mutatis mutandis from its isotropic counterpart [11,19]

$$
D_{\alpha}(h)=d_{H}\left(\left\{\mathbf{x} \mid h_{\alpha}(\mathbf{x})=h\right\}\right),
$$

where $h_{\alpha}(\mathbf{x})$ is defined as an anisotropic local regularity (or Hölder) exponent as

$h_{\alpha}(\mathbf{x})=\sup _{s}\left\{s ;\left|X_{\alpha_{0}, H_{0}, \lambda_{0}}(\mathbf{y})-P_{\mathbf{x}}(\mathbf{y})\right| \leq C\|\mathbf{y}-\mathbf{x}\|_{2, \alpha}^{s}\right\}$

with $P_{\mathbf{x}}(\mathbf{y})$ a polynomial of the form

$$
P\left(y_{1}, y_{2}\right)=\sum_{\left(\beta_{1}, \beta_{2}\right) \in \mathbb{N}^{2}} a_{\beta_{1}, \beta_{2}} y_{1}^{\beta_{1}} y_{2}^{\beta_{2}} .
$$

Scale-free properties. Based on the theoretical results obtained for isotropic multifractal fields in [13] and anisotropic self-similar processes in [17], one can naturally conjecture that the scale-free properties of $X_{\alpha_{0}, H_{0}, \lambda_{0}}(\mathbf{x})$ are wellcharacterized by its anisotropic multifractal spectrum taken in the nominal anisotropy direction $\alpha \equiv \alpha_{0}$ (with $c_{1}=$ $c_{1}\left(\alpha_{0}\right)=H_{0}-\lambda^{2}$ and $\left.c_{2}=c_{2}\left(\alpha_{0}\right)=\lambda^{2}\right)$ :

$$
D_{\alpha_{0}}(h)=2-\left(h-c_{1}\right)^{2} / c_{2} \text {. }
$$

\section{HYPERBOLIC WAVELET LEADERS}

Hyperbolic wavelet transform. The definition of 2D-DWT coefficients, $d_{X}(\boldsymbol{j}, \mathbf{k})=\langle X(\mathbf{x})| 2^{-2 j} \psi\left(2^{-j} \mathbf{x}-\mathbf{k}\right\rangle$, relies on the use of one same dilation factor $2^{j}$ for both directions, with the mother wavelet written as $\psi\left(x_{1}, x_{2}\right)=\psi_{0}\left(x_{1}\right) \psi_{0}\left(x_{2}\right)$, cf. e.g., [12]. In contrast, the hyperbolic wavelet transform (HWT) [16] is defined using two independent dilations factors $\left(2^{j_{1}}, 2^{j_{2}}\right)$ for the $x_{1}$ and $x_{2}$ coordinates

$$
\begin{aligned}
& d_{X}(\boldsymbol{j}, \mathbf{k})= \\
& \left\langle X\left(x_{1}, x_{2}\right) \mid 2^{-j_{1}} \psi\left(2^{-j_{1}} x_{1}-k_{1}\right) 2^{-j_{2}} \psi\left(2^{-j_{2}} x_{2}-k_{2}\right)\right\rangle
\end{aligned}
$$

where $\boldsymbol{j}=\left(j_{1}, j_{2}\right)$. It was shown in $[17,20,21]$ that it is precisely the use of these two independent dilation factors in the analysis that permits to disentangle self-similarity from anisotropy. HWT coefficients can be efficiently computed using alternate iterations of the classical $1 \mathrm{D}$ and $2 \mathrm{D}$ pyramidal algorithms underlying the DWT.

Hyperbolic wavelet leaders. To extend the anisotropy resolving property of HWT to multifractal analysis, we define hyperbolic wavelet leaders [21] in analogy with the $2 D-D W T$ leaders for isotropic multifractality proposed in [11]. Let $\beta>0$ and let $\lambda_{\boldsymbol{j}}(\mathbf{k})$ denote hyperbolic dyadic rectangles [21]

$$
\left.\left.\left.\left.\lambda_{j}(\mathbf{k})=\right] \frac{k_{1}}{2^{j_{1}}}, \frac{k_{1}+1}{2^{j_{1}}}\right] \times\right] \frac{k_{2}}{2^{j_{2}}}, \frac{k_{2}+1}{2^{j_{2}}}\right],
$$

Let $9 \lambda_{\boldsymbol{j}}(\mathbf{k})$ denote the union of $3^{2}$ such rectangles:

$$
\left.\left.\left.\left.9 \lambda_{j}(\mathbf{k})=\right] \frac{2^{j_{1}} k_{1}-1}{2^{j_{1}}}, \frac{2^{j_{1}} k_{1}+2}{2^{j_{1}}}\right] \times\right] \frac{2^{j_{2}} k_{2}-1}{2^{j_{2}}}, \frac{2^{j_{2}} k_{2}+2}{2^{j_{2}}}\right] .
$$

Hyperbolic wavelet leaders at scales $\boldsymbol{j}$ and location $\mathrm{k}$ are defined as local suprema of the HWT coefficients in a spatial neighborhood across finer scales $j_{1}^{\prime} \leq j_{1}$ et $j_{2}^{\prime} \leq j_{2}$ :

$$
L_{X}^{(\beta)}(\boldsymbol{j}, \mathbf{k})=\sup _{\boldsymbol{j}^{\prime}, \mathbf{k}^{\prime} \subset 9 \lambda_{\boldsymbol{j}}(\mathbf{k})} 2^{\frac{\left(j_{1}+j_{2}\right) \beta}{2}}\left|d_{X}\left(\boldsymbol{j}^{\prime}, \mathbf{k}^{\prime}\right)\right| .
$$


The interest of using leaders stems from the fact that it can be shown that when $X$ has an anisotropic local regularity exponent $h_{\alpha_{0}}\left(\mathbf{x}_{0}\right)>0$ at location $\mathbf{x}_{0}$, then $[17,21]$ :

$$
L_{X}(\boldsymbol{j}, \mathbf{k}) \leq 2^{\left(h_{\alpha_{0}}+\beta\right)\left(\mathbf{x}_{0}\right) \max \left(\frac{j_{1}}{\alpha_{0}}, \frac{j_{2}}{\left(2-\alpha_{0}\right)}\right)}
$$

which paves the way towards the estimation of $D_{\alpha_{0}}$. Here, the parameter $\beta$ permits to ensure that the process $X$ has enough regularity for the corner stone Eq. (8) to hold, cf., [11,21].

Anistropic multifractal spectrum estimation. To emphasize the targeted anisotropic nature of the analysis, we make use of relabelled scales $\boldsymbol{j}_{\alpha}(j)=(\alpha j,(2-\alpha) j)$, with $0<$ $\alpha<2$ the analysis anisotropy angle. Elaborating by analogy on calculations achieved independently for anisotropic Gaussian self-similar process [17] and isotropic multifractal processes [13], Eq. (8) above enables to conjecture that

$$
\begin{aligned}
\mathbb{E}\left(\left|L_{X}(\alpha j,(2-\alpha) j, \mathbf{k})\right|^{q}\right) \approx \\
2^{\frac{j}{\alpha}\left(q-\left(\tau_{\alpha_{0}}(q)+(1+\beta) q\right) \max \left(\frac{\alpha}{\alpha_{0}}, \frac{2-\alpha}{2-\alpha_{0}}\right)\right),}
\end{aligned}
$$

where $\tau_{\alpha_{0}}(q)$ denotes the Legendre transform of $D_{\alpha_{0}}$ [11]. The quantity $\tau(q, \alpha)=\left(q-\left(\tau_{\alpha_{0}}(q)+(1+\beta) q\right) \max \left(\frac{\alpha}{\alpha_{0}}, \frac{2-\alpha}{2-\alpha_{0}}\right)\right)$ is further maximal when $\alpha=\alpha_{0}$, which motivates the proposed estimation procedure.

The estimation of the multifractal spectrum in isotropic multifractal analysis can be based on several procedures (cf. e.g., [11] for reviews). We follow here a parametric strategy targeting the direct estimation of $c_{1}$ and $c_{2}$ in Eq. (5) [11], based on cumulants of the log hyperbolic wavelet leaders:

$$
\begin{aligned}
& C_{1}\left(\boldsymbol{j}_{\alpha}\right)=\frac{1}{n_{\boldsymbol{j}_{\alpha}}} \sum_{\mathbf{k} \in \mathbb{Z}^{2}}\left[\log L_{X}^{(\beta)}\left(\boldsymbol{j}_{\alpha}, \mathbf{k}\right)\right], \\
& C_{2}\left(\boldsymbol{j}_{\alpha}\right)=\frac{1}{n_{\boldsymbol{j}_{\alpha}}} \sum_{\mathbf{k} \in \mathbb{Z}^{2}}\left[\log L_{X}^{(\beta)}\left(\boldsymbol{j}_{\alpha}, \mathbf{k}\right)\right]^{2}-\left[C_{1}\left(\boldsymbol{j}_{\alpha}\right)\right]^{2},
\end{aligned}
$$

where $n_{\boldsymbol{j}_{\alpha}}$ denotes to the number of hyperbolic wavelet leaders available at scales $j_{\alpha}$. For the anisotropic multifractal texture defined in Eq. (1), Eq. (9) leads to

$$
\begin{aligned}
& C_{1}(\alpha j,(2-\alpha) j) \sim c_{1}(\alpha) \ln 2^{j} \\
& C_{2}(\alpha j,(2-\alpha) j) \sim-c_{2}(\alpha) \ln 2^{j}
\end{aligned}
$$

and estimates $\hat{c}_{p}(\alpha)$ can thus be obtained by linear regressions of $C_{p}(\alpha j,(2-\alpha) j)$ versus $j$, cf. $[11,21]$. The parameters $\left(\alpha_{0}, \hat{c}_{1}, \hat{c}_{2}\right)$ are then obtained as

$$
\hat{\alpha}_{c_{p}}=\operatorname{argmax}_{\alpha} c_{p}(\alpha), \quad \hat{c}_{p}=c_{p}\left(\hat{\alpha}_{c_{p}}\right)
$$

and are straightforward to translate into estimates for $H_{0}$ and $\lambda_{0}$. Note that the use of the analysis angle $\alpha=1$ in this procedure essentially amounts to recovering the classical 2DDWT leaders proposed in [11].

\section{PERFORMANCE ASSESSMENT}

Synthetic textures and performance assessment. To assess the relevance and performance of the proposed model and estimation procedure, three different settings for the parameters
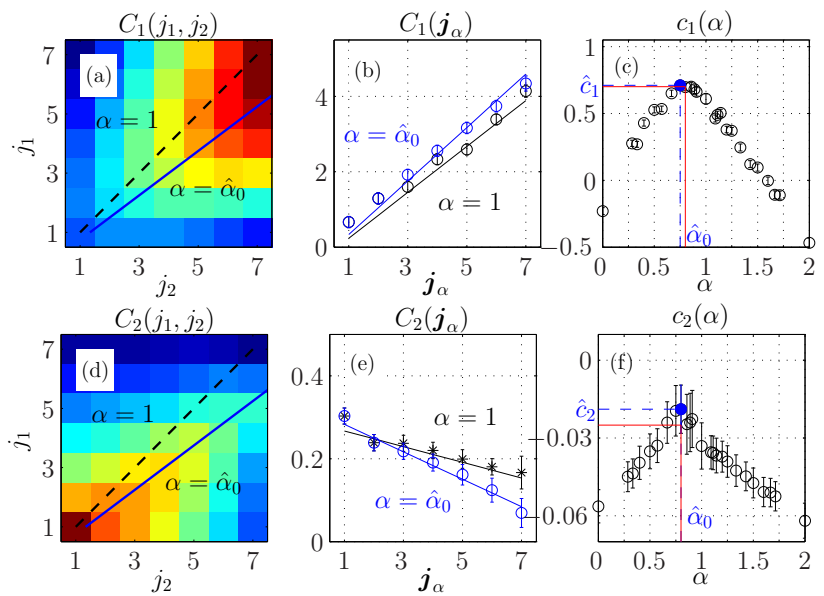

Fig. 1. Anisotropic multifractal textures. Plots of $C_{p}$ and $c_{p}$ for $p=1$ (top row) and $p=2$ (bottom row). Left column: $C_{p}\left(j_{1}, j_{2}\right)$ surface with the dashed black and the blue solid lines indicating the isotropic and the estimated anisotropic directions $\alpha \equiv 1$ and $\alpha=\hat{\alpha}_{0}$, respectively. Middle column: $C_{p}\left(\boldsymbol{j}_{\alpha}(j)\right)$ as functions of $j$, for $\alpha \equiv 1$ (black line with ' $*$ ') and for $\alpha=\hat{\alpha}_{0}$ (blue line with 'o'). Right column: $c_{p}(\alpha)$ as functions of $\alpha$ (average and standard deviations across realizations). The location and value at the maximum of $c_{p}(\alpha)$ permits to estimate $\alpha_{0}$ and $c_{p}$. The theoretical values for $\left(\alpha_{0}, c_{2}\right)$ are shown with red lines.

$\left(\alpha_{0}, H_{0}, \lambda_{0}\right)$ are studied: a multifractal anisotropic texture with $(0.8,0.7, \sqrt{0.025})$; a multifractal isotropic texture with $(1,0.7, \sqrt{0.025})$; a self-similar (non multifractal) anisotropic texture (OSGRF) with $(0.8,0.7,0)$. For each parameter setting, $N=100$ independent copies of size $1024 \times 1024$ are generated with MATLAB routines designed by ourselves (and available upon request). Samples for the three cases are shown in Fig. 4. Daubechies least asymmetric mother wavelet with 3 vanishing moments [12] are used to compute the hyperbolic wavelet leaders for $j_{1}$ and $j_{2}$ ranging from 1 to 7 . Eqs. (12) and (13)) are used to estimate $c_{1}(\alpha)$ and $c_{2}(\alpha)$ by linear regressions. Estimation of $c_{1}(\alpha)$ and $c_{2}(\alpha)$ and of $\alpha_{0}, c_{1}$ and $c_{2}$ is then performed on each realization independently. Average results for the three cases are plotted in Figs. 1 - 3, respectively, and further quantified in Tab. 1

Performance assessment. Fig. 1 shows that for anisotropic multifractal textures, both functions $c_{1}(\alpha)$ and $c_{2}(\alpha)$ display clear maxima located at the same $\alpha$, thus yielding consistant estimates $\hat{\alpha}_{0} \simeq 0.79$ of $\alpha_{0}$. Because it is not granted by construction, such consistency in the estimation of $\alpha_{0}$ is a satisfactory outcome validating the relevance of the procedure. The values for $c_{p}$ are also satisfactorily estimated, with small biases and variances. For isotropic multifractal textures (cf., Fig. 2), $\hat{\alpha}_{0} \simeq 1$ consistently for both $p=1$ and $p=2$, indicating the absence of anisotropy. For that case, the hyperbolic wavelet leaders essentially amounts to the classical 2D-DWT leaders, yet without having been selected a priori. Moreover, 

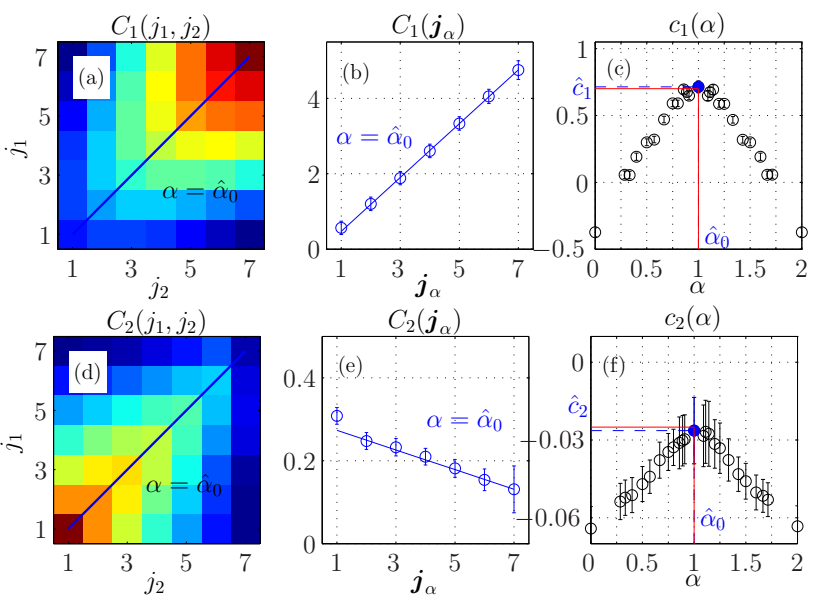

Fig. 2. Isotropic multifractal texture. Legend as in Fig. 1.
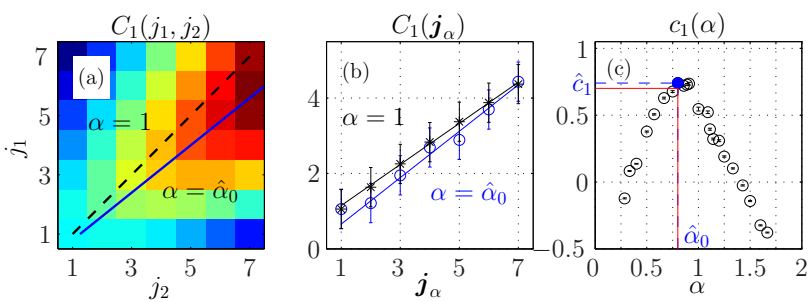

$C_{2}\left(j_{1}, j_{2}\right)$
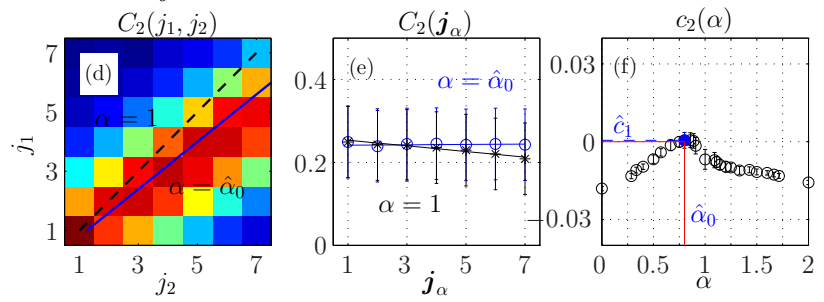

Fig. 3. Anisotropic non multifractal texture. Legend as in Fig. 1.
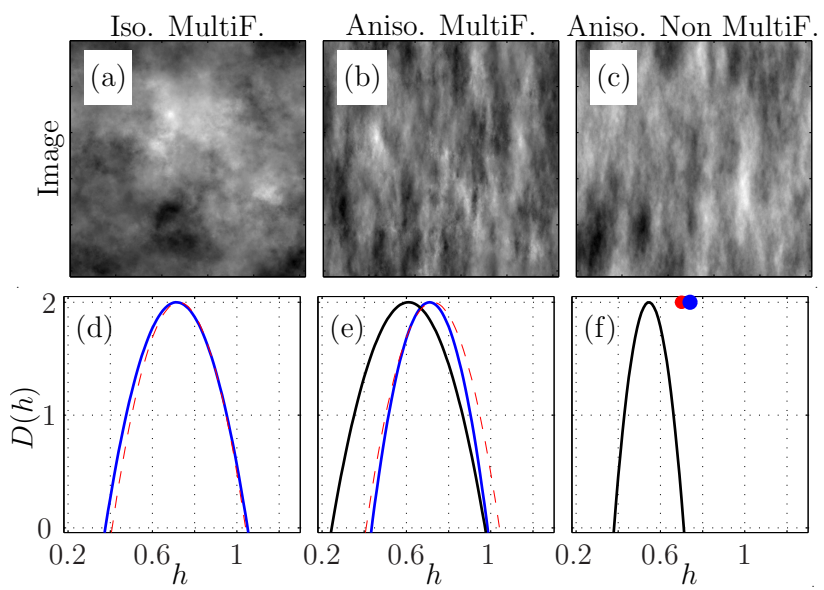

Fig. 4. Hyperbolic versus classical wavelet leaders multifractal textures. Top row: sample textures. Bottom row: multifractal spectra estimated using 2D-DWT classical leaders, i.e., $\alpha=1$ (black) and hyperbolic wavelet leaders, with $\alpha=\hat{\alpha}_{0}$ (blue) compared to the theoretical spectrum (red).

\begin{tabular}{|c||c|c|c|}
\hline & Aniso. MultiF. & Iso. MultiF. & Aniso. Non MultiF. \\
\hline \hline$\hat{c}_{1}$ & $0.099(0.029)$ & $0.013(0.044)$ & $0.039(0.007)$ \\
\hline$\hat{\alpha}_{c_{1}}$ & $0.017(0.049)$ & $0.008(0.085)$ & $0.041(0.053)$ \\
\hline$\hat{c}_{2}$ & $0.006(0.009)$ & $0.002(0.012)$ & $0.001(0.003)$ \\
\hline$\hat{\alpha}_{c_{2}}$ & $0.007(0.098)$ & $0.021(0.125)$ & $0.034(0.080)$ \\
\hline
\end{tabular}

Table 1. Bias and standard deviation for estimates.

Fig. 3 indicates that for anisotropic self-similar (non multifractal) textures, $c_{1}(\alpha)$ also displays a clear maximum whose location $\hat{\alpha}_{0} \simeq 0.83$ nicely estimates $\alpha_{0}$ and whose value $\hat{c}_{1}=c_{1}\left(\alpha=\hat{\alpha}_{0}\right) \simeq 0.73$ nicely matches $H_{0}$. Conversely, the function $c_{2}(\alpha)$ remains essentially flat at 0 , showing no multifractality $\hat{c}_{2} \simeq 0$, with a slight practical maximum with same location as that of $c_{1}(\alpha)$, a satisfactory practical outcome of the procedure. The performance for all three parameter settings are further quantified in Tab. 1. The results indicate that the proposed multifractal anisotropic analysis procedure consistently yields satisfactory estimates with small biases and variances for all textures, be they anisotropic or not, multifractal or not.

Finally, Fig. 4 compares the multifractal spectra obtained using hyperbolic wavelet leaders to those obtained using classical 2D-DWT leaders. As expected, for isotropic textures, both methods yield equivalent estimates that match well the theoretical multifractal spectrum. Yet, for the anisotropic multifractal and the anisotropic non multifractal textures, 2D-DWT leaders fail to provide relevant estimates of the multifractal spectra and significantly overestimate the multifractality of the texture. In addition, note that it would not be possible for practitioners to detect that their 2D-DWT leaders based analysis is fooled by anisotropy. In contrast, estimates obtained with the proposed hyperbolic wavelet leaders remain excellent, which further underlines their benefits.

\section{CONCLUSION AND FUTURE WORKS}

A double contribution has been proposed here: An operational anisotropic multifractal texture has been defined, which, to the best of our knowledge, was so far not available in the literature; A procedure for the anisotropic multifractal analysis has been devised, which again is seen as an original contribution. Elaborating on the present work, the statistical properties of the proposed anisotropic multifractal texture model will be further assessed in a more theoretical framework. Accordingly, the properties of its hyperbolic wavelet leaders will be further consolidated theoretically. At the practical level, we believe that the proposed analysis tool is now ready and sound enough for application to real-world textures, where both anisotropy and scale free properties are likely to be relevant together, and will thus permit an estimation of multifractality not fooled by anisotropy. 


\section{REFERENCES}

[1] D. Schertzer and S. Lovejoy, "Physically based rain and cloud modeling by anisotropic, multiplicative turbulent cascades," J. Geophys. Res., vol. 92, pp. 9693-9714, 1987.

[2] A. Arneodo, N. Decoster, and S. G. Roux, "A waveletbased method for multifractal image analysis. i. methodology and test applications on isotropic and anisotropic random rough surfaces," European Physical Journal B, vol. 15 , no. 3, pp. 567-600, June 2000.

[3] C. L. Benhamou, S. Poupon, E. Lespessailles, S. Loiseau, R. Jennane, V. Siroux, W. J. Ohley, and L. Pothuaud, "Fractal analysis of radiographic trabecular bone texture and bone mineral density: two complementary parameters related to osteoporotic fractures," $J$. Bone Miner. Res., vol. 16, no. 4, pp. 697-704, 2001.

[4] R. Lopes and N. Betrouni, "Fractal and multifractal analysis: A review," Medical Image Analysis, vol. 13, pp. 634-649, 2009.

[5] R. Lopes, P. Dubois, I. Bhouri, M.H. Bedoui, S. Maouche, and N. Betrouni, "Local fractal and multifractal features for volumic texture characterization," Pattern Recognition, vol. 44, no. 8, pp. 1690-1697, 2011.

[6] P. Abry, S. Jaffard, and H. Wendt, "When Van Gogh meets Mandelbrot: Multifractal classification of painting's texture," Signal Proces., vol. 93, no. 3, pp. 554$572,2013$.

[7] N. Pustelnik, H. Wendt, and P. Abry, "Local regularity for texture segmentation: Combining wavelet leaders and proximal minimization," in in Proc. of IEEE Int. Conf. on Acoustics, Speech and Signal Processing (ICASSP), Vancouver, Canada, May 2013.

[8] N. Pustelnik, P. Abry, H. Wendt, and N. Dobigeon, "Inverse problem formulation for regularity estimation in images," in Proc. IEEE Int. Conf. Image Proc. (ICIP), Paris, France, Oct. 2014.

[9] P. Abry, S. G. Roux, H. Wendt, P. Messier, A. G. Klein, N. Tremblay, P. Borgnat, S. Jaffard, B. Vedel, J. Coddington, and L. Daffner, "Multiscale anisotropic texture analysis and classification of photographic prints: Art scholarship meets image processing algorithms," IEEE Signal Proc. Mag., vol. 32, no. 4, pp. 18-27, July 2015.

[10] G. Samorodnitsky and M. Taqqu, Stable non-Gaussian random processes, Chapman and Hall, New York, 1994.
[11] H. Wendt, S.G. Roux, S. Jaffard, and P. Abry, "Wavelet leaders and bootstrap for multifractal analysis of images," Signal Processing, vol. 89, no. 6, pp. 1100-1114, June 2009.

[12] S. Mallat, A Wavelet Tour of Signal Processing, Academic Press, San Diego, CA, 1998.

[13] R. Robert and V. Vargas, "Hydrodynamic turbulence and intermittent random fields," Communications In Mathematical Physics, vol. 284, no. 3, pp. 649-673, Dec. 2008.

[14] B. B. Mandelbrot, "Intermittent turbulence in selfsimilar cascades: divergence of high moments and dimension of the carrier," J. Fluid Mech., vol. 62, pp. 331-358, 1974.

[15] H. Biermé, M.M. Meerschaert, and H.P. Scheffler, "Operator scaling stable random fields.," Stoch. Proc. Appl., vol. 117, no. 3, pp. 312-332, 2009.

[16] R. A. DeVore, S. V. Konyagin, and V. N. Temlyakov, "Hyperbolic wavelet approximation," Constr. Approx., vol. 14, pp. 1-26, 1998.

[17] S.G. Roux, M. Clausel, B. Vedel, S. Jaffard, and P. Abry, "Self-similar anisotropic texture analysis: The hyperbolic wavelet transform contribution," IEEE Trans. Image Proces., vol. 22, no. 11, pp. 4353-4363, 2013.

[18] E. Bacry, J. Delour, and J. R. Muzy, "Multifractal random walk," Phys. Rev. E, vol. 64, no. 2, pp. 026103, 2001.

[19] M Ben Slimane and H Ben Braiek, "Baire generic results for the anisotropic multifractal formalism," Revista Matemática Complutense, vol. 29, no. 1, pp. 127-167, 2016.

[20] S.G. Roux, M. Clausel, B. Vedel, S. Jaffard, and P. Abry, "Transformé hyperbolique en ondelettes $2 \mathrm{~d}$ pour la caractérisation d'images autosimilaires anisotropes," in XXIII colloque sur le Traitement du Signal et des Images GRETSI, Bordeaux, France, 5-8 Sep. 2011.

[21] P. Abry, M. Clausel, S. Jaffard, S.G. Roux, and B. Vedel, "Hyperbolic wavelet transform: an efficient tool for multifractal analysis of anisotropic textures," Revista Matemática Iberoamericana, vol. 1, 2015. 